\title{
Development of the Drone with the Function of Flight and Vehicle to Save Power Consumption
}

\author{
Ryunosuke Tadoh ${ }^{1 *}$, Huimin $\mathrm{Lu}^{2}$, Shiyuan Yang ${ }^{1}$, Seiichi Serikawa ${ }^{1}$ \\ ${ }^{1}$ Department of Electrical and Electronics Engineering, Kyushu Institute of Technology, Japan \\ ${ }^{2}$ Department of Mechanical and Control Engineering, Kyushu Institute of Technology, Japan \\ *Corresponding Author: n108071r@mail.kyutech.jp
}

\begin{abstract}
In recent years, the drone technology is developing rapidly. Drone is going to be used in many places, e.g., photography, delivery and agriculture. Due to the development of drone technology, we must reduce energy consumption, and realize high range movement. However, the drone can fly only about 20 minutes at once. In order to solve this limitation, we develop a new type of drone. The drone with the function of flight and vehicle can move less power consumption, and it lead high range of mobility to drone.
\end{abstract}

Keywords: Battery, Drone, Energy.

\section{Introduction}

Because of faster control signal, smaller motor and lighter computer, the drone technology has come rapid development. Mainly, the drones has potential to work in the field of photography, delivery and agriculture, and around the world countries and companies have high hopes for its potential. The BI intelligence expects that drone market is going to be reached at the \$ 12 billion until 2024 in the worldwide ${ }^{(1)}$.

Japan doesn't have much large scale land compared with the other countries. It is possible that we can get benefit expanding the use of airspace. At the public-private Association for environment improvement of small unmanned air vehicle, we are aiming to realize delivering service by drone till 2019. In addition, aiming to realize a society of that autonomous drones are active in areas including cities from the 2020s ${ }^{(2)}$. An experiment was held on $11 / 2016$ at the Chiba city which is the national strategic zone drone flied 700 meters over the sea and the book were reached at goal ${ }^{(3)}$.

It is necessary that investment for technology by companies due to expand drone use, but there are some restrictions. For example, drone flies only 20 30min and cannot go far away, it be required circumvent around the no-fly zone, and it isn't safe in the strong wind. So, we need to develop drone that flying more distant and more efficiently ${ }^{(4)}$.

There are many previous studies. First one is that car has only wheels and does not have motor but it move with wind power by propellers. However, this system has a drawback. This drone cannot move minutely. One of the benefit function of flight and vehicle is that change position in detail, but it is so difficult to do that by wind power. The second one is that has caterpillar instead of wheels. However, caterpillar system cost high energy ${ }^{(5)}$.

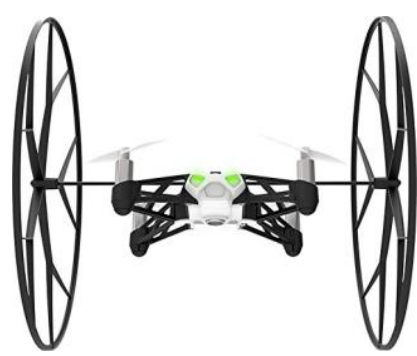

Fig. 1. Wind power

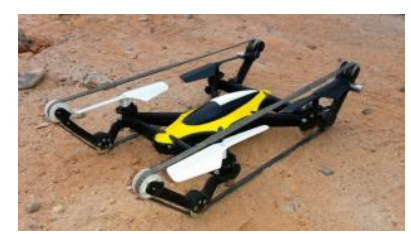

Fig. 1. Caterpillar

So, we intended to develop the drone with motor car, and reduce power consumption. Furthermore, we compensate the shortcoming of flying by driving system. 


\section{System Configuration}

\subsection{Multicopter}

There are various drones like airplane type or helicopter type. Multicopter is one type of the Unmanned Aerial Vehicles (UAVs). Its power source is a battery. Generally, it has four or more DC motors.

Table 1. Types of multicopter

\begin{tabular}{|c|c|c|c|c|}
\hline name & Number of rotors & Stability & Weight & Maintenance \\
\hline Quadcopter & 4 & Low & Light & Few \\
\hline Hexacopter & 6 & $\mid$ & $\mid$ & $\mid$ \\
\hline Octocopter & 8 & High & Heavy & Much \\
\hline
\end{tabular}

We use quadcopter, "Parrot AR.Drone 2.0 (6)" which is propelled by four rotors.

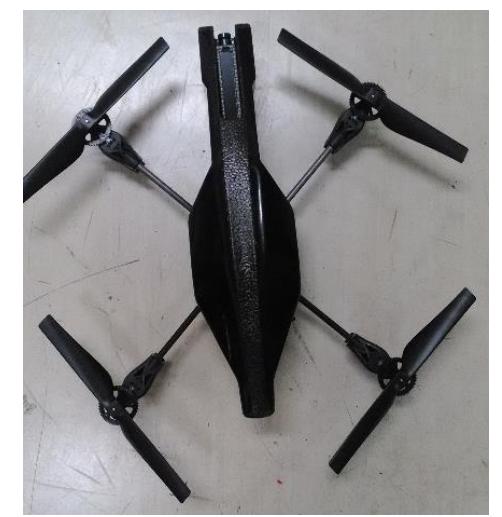

Fig. 3. Parrot AR.Drone 2.0

\subsection{Constitution of Drone with Motor Car}

In this research, we develop the drone with motor car. And, switching between flying and driving, measuring of each power consumption. We use the drone which communicated with Wi-Fi. And we control it from iOS application.

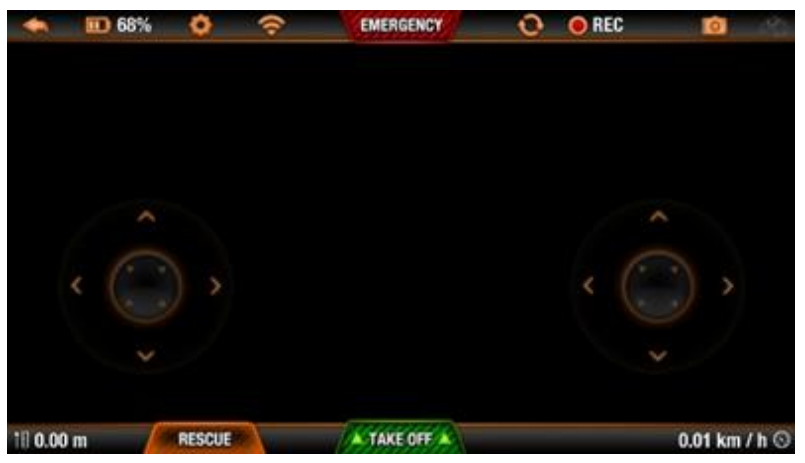

Fig. 4. AR.Drone application
In addition, attached "Raspberry Pi 2 model B" for control the motor car. Raspberry $\mathrm{Pi}^{(7)}$ has forty GPIO pins.

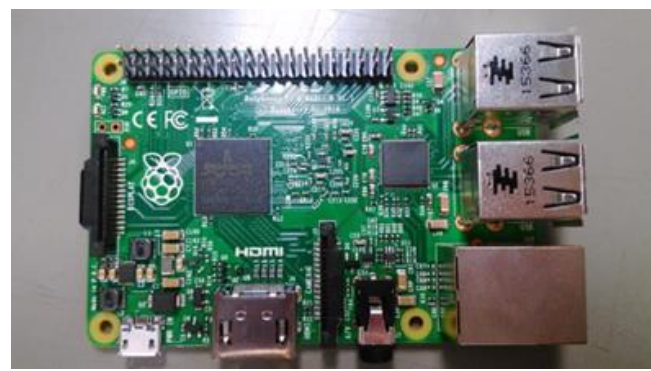

Fig. 5. Raspberry Pi 2 model B

We make a circuit with a motor driver to control motor car by Raspberry Pi. Raspberry Pi with a Wi-Fi dongle connect to the drone Wi-Fi. Plus, the laptop for control GPIO pins connect to the drone too. This means that Raspberry Pi and laptop exist on the same network. In that state, we used an application called WebIOPi ${ }^{(8)}$ to link both GPIO pin and laptop. WebIOPi is an application for accessing Raspberry Pi GPIO pins through a browser. It makes us to control motor car.

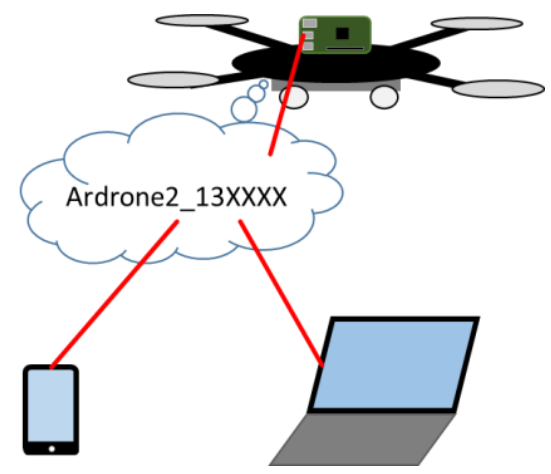

Fig. 6. System Configuration

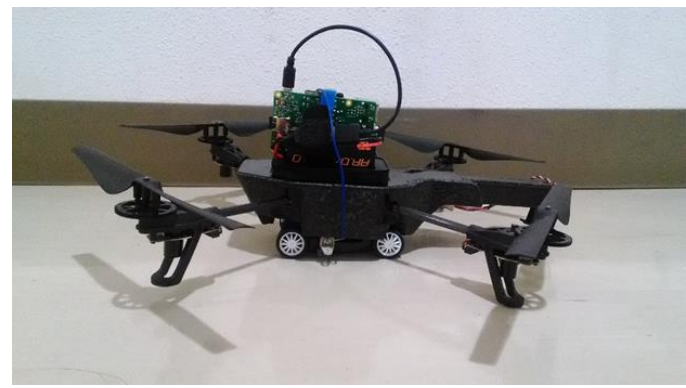

Fig. 7. Drone Car

\section{Experimental Results}

Operation of the drone is carried by the laptop and the smartphone. In particular, motor car is operated 
from the laptop, and drone is operated from the smartphone.

The payload of "Parrot AR.Drone 2.0" is $142 \mathrm{~g}$, and the weight of the whole system is $139 \mathrm{~g}$. So, the drone car fly without problem. Furthermore, switching of running and flying could be smoothly and that can be used for experiments.

First, we research the power consumption of flying. And it compared with the drone without car system.

Table 2. Flight experiment

\begin{tabular}{|c|c|c|}
\hline System & Flight Time(s) & Consumption(W) \\
\hline Before & 630 & 105 \\
\hline After & 625 & 106 \\
\hline
\end{tabular}

As shown in this table, it is found that the power consumption increased $1 \mathrm{~W}$ by installation of the system.

Next, we research the power consumption of running. We checked current value from the battery during it runs.

Table 3. Running experiment

\begin{tabular}{|c|c|}
\hline Times & Current $(\mathrm{mA})$ \\
\hline 1 & 442 \\
\hline 2 & 447 \\
\hline 3 & 443 \\
\hline 4 & 447 \\
\hline 5 & 446 \\
\hline 6 & 442 \\
\hline 7 & 442 \\
\hline 8 & 441 \\
\hline 9 & 442 \\
\hline 10 & 445 \\
\hline Average & 443.5 \\
\hline
\end{tabular}

The average current value is $443.5 \mathrm{~mA}$. Since the output voltage of the battery is $12.3 \mathrm{~V}$, the average power consumption is $5.5 \mathrm{~W}$.

The above results are summarized as follows. Drone before system installation (flying): $105 \mathrm{~W}$ Drone after installing the system (flying): $106 \mathrm{~W}$ Drone after installing the system (running): $5.5 \mathrm{~W}$

\section{Conclusion}

From the results of the experiment, we find that the power consumption is very low when running the drone car compared with the case of flying.

As a future task, we are planning to equip with $\mathrm{AI}{ }^{(9,10)}$. It realize that a system switches autonomously between running and flight according to the environment. Also we are planning to update its hardware sturdier and improve position control accurately.

\section{Acknowledgment}

This work was supported by JSPS KAKENHI (No.15F15077), Leading Initiative for Excellent Young Researcher (LEADER) of Ministry of Education, Culture, Sports, Science and Technology-Japan (16809746), Research Fund of Chinese Academy of Sciences (No.MGE2015KG02), Research Fund of State Key Laboratory of Marine Geology in Tongji University (MGK1608), and Research Fund of State Key Laboratory of Ocean Engineering in Shanghai Jiaotong University $(1315 ; 1510)$.

\section{References}

(1) Akihito Kobayashi, : "The singular impact of drone business A new market opened by small unmanned aircraft ", Asahi Shimbun Publication, 2015, July

(2) Ministry of Economy Trade and Industry : "Road map of utilization of small unmanned aircraft and technology development", 2016, April

(3) Yomiuri Online : "Examination of the creation of Zero-based Special Zone for drone delivery experiment", 2016, December

(4) NTT data, Masato Kawamura, Hiroshi Ootuka, Yuusuke Kobayashi, Takeshi Koyama, Tomoya Miyazaki, Yuuki Ishiguro, Kouhei Kozima : "IoT / Sensor mechanism and utilization seen by picture" 2015

(5) Izumi Nakagawa, Junko Takeda, Whiteman Philip : "The aircraft book", 2015

(6) Parrot AR. Drone 2.0, https://www.parrot.com/fr/drones/parrot-ardrone-20-eli te-\%C3\%A9dition\#parrot-ardrone-20-elite-edition

(7) Raspberry Pi, https://www.raspberrypi.org/

(8) The Raspberry $\mathrm{Pi}$ Internet of Things Toolkit, http://webiopi.trouch.com/

(9) Y. Li, H. Lu, J. Li, X. Li, Y. Li, S. Serikawa, "Underwater image de-scattering and classification by deep neural network," Computers and Electrical 
Engineering, vol.54, pp.68-77, 2016.

(10) S. Mu, C. Zhou, H. Lu, "Neural network based on-line stabilization for humanoid robots," In Proceedings of 2016 International Symposium on Computer, Consumer and Control, pp.140-143, 2016. 